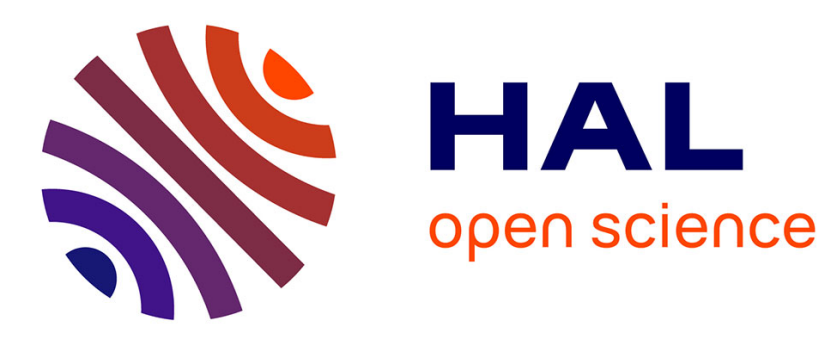

\title{
Vers l'intégration des compétences dans le pilotage des performances de l'entreprise
}

\author{
Xavier Boucher, Patrick Burlat
}

\section{To cite this version:}

Xavier Boucher, Patrick Burlat. Vers l'intégration des compétences dans le pilotage des performances de l'entreprise. Journal Européen des Systèmes Automatisés (JESA), 2003, Vol. 37 (3), pp. 363-390. 10.3166/jesa.37.363-390 . emse-00755899

\section{HAL Id: emse-00755899 https://hal-emse.ccsd.cnrs.fr/emse-00755899}

Submitted on 26 Nov 2021

HAL is a multi-disciplinary open access archive for the deposit and dissemination of scientific research documents, whether they are published or not. The documents may come from teaching and research institutions in France or abroad, or from public or private research centers.
L'archive ouverte pluridisciplinaire HAL, est destinée au dépôt et à la diffusion de documents scientifiques de niveau recherche, publiés ou non, émanant des établissements d'enseignement et de recherche français ou étrangers, des laboratoires publics ou privés. 


\title{
Vers l'intégration des compétences dans le pilotage des performances de l'entreprise
}

\author{
Xavier Boucher * Patrick Burlat ${ }^{*}$ \\ $*$ \\ Ecole Nationale Supérieure des Mines de Saint-Etienne, \\ 158 cours Fauriel, F-42023 Saint-Etienne cedex, \\ boucher@emse.fr,burlat@emse.fr
}

RÉSUMÉ. Cet article propose une méthode de conception d'indicateurs de performance intégrant le concept de compétence dans le pilotage d'organisations industrielles. Les variables d'actions du pilotage sont explicitées à partir d'un modèle reliant les compétences aux situations professionnelles, aux acteurs et aux ressources industrielles. La stratégie est exprimée sous forme de macro-compétences et la performance est déclinée en terme d'efficacité, de pertinence et d'efficience. Des indicateurs de résultat sont alors calculés à partir de la théorie des sous-ensembles flous. Un tableau de bord destiné au pilotage opérationnel relie ces indicateurs avec les variables d'actions. En final, un exemple industriel est présenté comme illustration de cette démarche.

\begin{abstract}
This article describes a method to design performance indicators including competencies, so as to control industrial organizations. The variables required to control an organization are based on a model tying competencies with professional situations, actors and industrial resources. The strategy of a firm is delineated with macro-competencies, and its performance is divided into efficaciousness, pertinence and efficiency. Indicators of results are calculated thanks to the fuzzy sets theory. A score card for operational control links these indicators with variables of control. Eventually, an industrial example is given to illustrate this method.
\end{abstract}

MOTS-CLÉS : Performance, compétence, pilotage, sous-ensembles flous, indicateurs, tableau de bord.

KEY WORDS: Performance, competence, control, fuzzy sets, indicators, score card. 


\section{Introduction}

Dans le domaine des sciences de gestion, Prahalad et al. argumentaient au début des années 90 qu'il était désormais nécessaire pour les entreprises de se considérer comme des «systèmes de compétences » et de développer une stratégie industrielle s'appuyant sur la gestion des noyaux de compétences ('core competences') [PRA 92]. Cette publication cristallisait une prise de conscience de la communauté scientifique sur la gestion des compétences comme levier d'action pour maîtriser la complexité des Systèmes de Production (SP) et allait donner naissance à de nouveaux développements théoriques en sciences de gestion notamment avec l'approche basée sur les ressources et l'approche basée sur les compétences [QUE 00].

Cette thématique de recherche est actuellement largement relayée dans d'autres domaines scientifiques et tout particulièrement dans le génie industriel : la notion de compétence s'impose progressivement comme un point de vue indispensable pour la modélisation en entreprise [VER 99]. Différents travaux permettent aujourd'hui d'intégrer la dimension « compétence » dans des thématiques classiques du génie industriel [BOU 01]:

- développement de modèles formels pour l'intégration du concept de compétence dans la modélisation en entreprise [HAR 00], [DUL 01], [JIA 98], [SZE 00];

- prise en compte de la compétence comme l'un des paramètres à intégrer dans les processus de planification propres à la gestion de production [FRA 00], [GRA 00], [JIA 98] ;

- proposition de méthodes et outils pour favoriser la maîtrise des compétences collectives dans un contexte d'ingénierie concourante [BOU 99], [GAR 99], [RAU 01] ;

- intégration de la dimension « compétence» dans les méthodologies de réorganisation en entreprise [HAR 00a], [BER 01], [PER 00].

La compétence s'affirme donc comme l'un des paramètres importants de la performance en production et l'objectif de cet article est d'apporter une contribution pour intégrer de manière explicite et opérationnelle ce paramètre dans les systèmes d'évaluation (indicateurs de performance) des organisations, puis dans les mécanismes de pilotage (notion d'inducteur de performance).

Après avoir défini la problématique justifiant notre contribution (paragraphe 1), nous présenterons les éléments clé des systèmes d'indicateurs de performance à prendre en compte pour intégrer la dimension compétence (paragraphe 2). Puis, nous exposerons l'impact du concept de compétence sur la problématique classique de la performance (paragraphe 3), notamment en détaillant un modèle qualitatif de la compétence sur lequel s'appuient nos développements. Dans une seconde partie, nous développerons en deux temps notre contribution conceptuelle. Le paragraphe 4 proposera un modèle permettant de décrire et de décliner les «macro-compétences » d'une entreprise en établissant le lien entre les différents niveaux de pilotage de l'entreprise. Au paragraphe 5, nous utilisons ce modèle pour la construction de tableaux de bord de pilotage. Nous préciserons comment intégrer la compétence dans le triptyque classique de la performance (pertinence, efficacité, efficience [BES 95]), en proposant de nouveaux indicateurs notamment destinés à élaborer une vision prédictive du développement organisationnel. 
Pour terminer, nous détaillerons une étude de cas concernant une PME de la région Rhône Alpes. Outre la mise en œuvre du modèle descriptif des macrocompétences et des tableaux de bord de pilotage associés, cette étude de cas mettra en évidence certains leviers d'action ainsi que la possibilité de les hiérarchiser.

\section{Performance industrielle et indicateurs}

Le concept d'indicateur de performance a été défini par l'Association Française de Gestion Industrielle (AFGI) comme «une donnée quantifiée qui mesure l'efficacité de tout ou partie d'un processus ou d'un système par rapport à une norme, un plan ou un objectif qui aura été déterminé et accepté dans le cadre d'une stratégie d'ensemble » [GAL 90]. Selon cette définition, un indicateur de performance n'a de sens que par rapport à un objectif issu d'une stratégie industrielle. Dans cet article, c'est la stratégie d'apprentissage de l'entreprise qui servira de cadre pour orienter la conception d'un système d'indicateurs. Cette stratégie sera exprimée plus loin en terme d'acquisition, de maîtrise, et de développement de «macrocompétences ».

Nous représenterons la performance d'une organisation selon l'approche communément acceptée de P. L. Bescos qui explicite les concepts d'efficacité, d'efficience et de pertinence à partir du triplet (objectifs, résultats, moyens) [BES 95] :

Objectifs

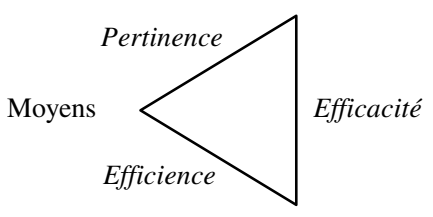

Résultats

Figure 1 : Efficacité, efficience et pertinence

Dans ce modèle, l'efficacité caractérise l'écart entre les objectifs à atteindre et les résultats obtenus. Elle mesure l'aptitude du système de pilotage à tenir les objectifs qui lui ont été assignés. La pertinence concerne l'adéquation entre moyens mis en œuvre et objectifs. Son évaluation pose la question du réalisme des moyens alloués par rapport aux objectifs visés. Enfin, l'efficience mesure le rendement du système par comparaison entre les moyens mis en œuvre et les résultats obtenus. Dans le cadre de cet article, nous considérerons que l'écart entre l'objectif de maîtrise de compétences par une organisation et le résultat effectivement obtenu s'explique par une inadéquation des moyens et/ou par une mauvaise mise en œuvre de ces moyens. En d'autres termes, nous considérerons qu'un problème d'efficacité provient d'un problème de pertinence, ou d'efficience, ou des deux à la fois. 
En conséquence, et dans l'optique de la construction d'un tableau de bord destiné au pilotage opérationnel des compétences, nous devrons identifier les inducteurs de performance $^{1}$ qui caractérisent les moyens et qui permettent d'influer sur les indicateurs d'efficience et de pertinence. Ces variables d'actions constitueront les leviers pour le pilotage du système de compétences d'une organisation. Elles sont explicitées dans les paragraphes suivants.

\section{Apport des compétences à la problématique de la performance}

\subsection{Problématique considérée}

La plupart des approches actuelles des tableaux de bord de performance s'appuient sur le point de vue selon lequel la performance d'une entreprise repose avant tout sur la maîtrise de ses processus de création de produits et de service. Cette approche de la performance est induite par les travaux précurseurs de Porter sur la chaîne de valeur [POR 93]. En opposition avec cet a priori, le modèle de gestion proposé par l'approche Ressource Based View situe la source de l'avantage concurrentiel d'une firme dans ses actifs internes, et notamment dans la construction de ses compétences [QUE 00]. Ces deux points de vue, plutôt qu'opposés nous semblent complémentaires et nous proposons dans cet article de considérer une entreprise comme un double système de production (figure 2) :

- l'entreprise est considérée comme un système de production de biens et de services ; de ce point de vue sa performance réside (de manière non exclusive) dans la maîtrise de ses processus de réalisation de valeur ;

- l'entreprise est considérée par ailleurs comme un système de production de compétences ; de ce point de vue sa compétitivité repose sur la maîtrise des processus de développement de compétence.

La figure 2 traduit la complémentarité entre ces deux approches de l'efficience (et non pas un simple parallélisme : la performance d'un système de production de biens ou de services est induite par le niveau de ses compétences, et la valeur ajoutée générée est en partie ré-investie (investissement immatériel en formation par exemple) pour améliorer le niveau des compétences. Par rapport à cette vision globale de la maîtrise des performances, nous positionnons notre contribution actuelle sur le développement du potentiel de réalisation de valeur dans une organisation, c'est à dire sur la maîtrise et la régulation du système de production de compétences.

\footnotetext{
${ }^{1}$ L'inducteur de performance, extension de la notion d'inducteur de coût [LOR 97], établit une relation causale entre une performance et un fait observable. Lorsqu'une intervention est possible sur la cause de la performance, (i. e. lorsque le fait est modifiable car accessible dans l'espace des décisions de l'entreprise), cette cause devient une variable d'action pour le pilotage de la performance. Ces variables d'actions constituent donc la base sur laquelle sont appliqués les plans d'actions lors de démarches d'amélioration de performances.
} 


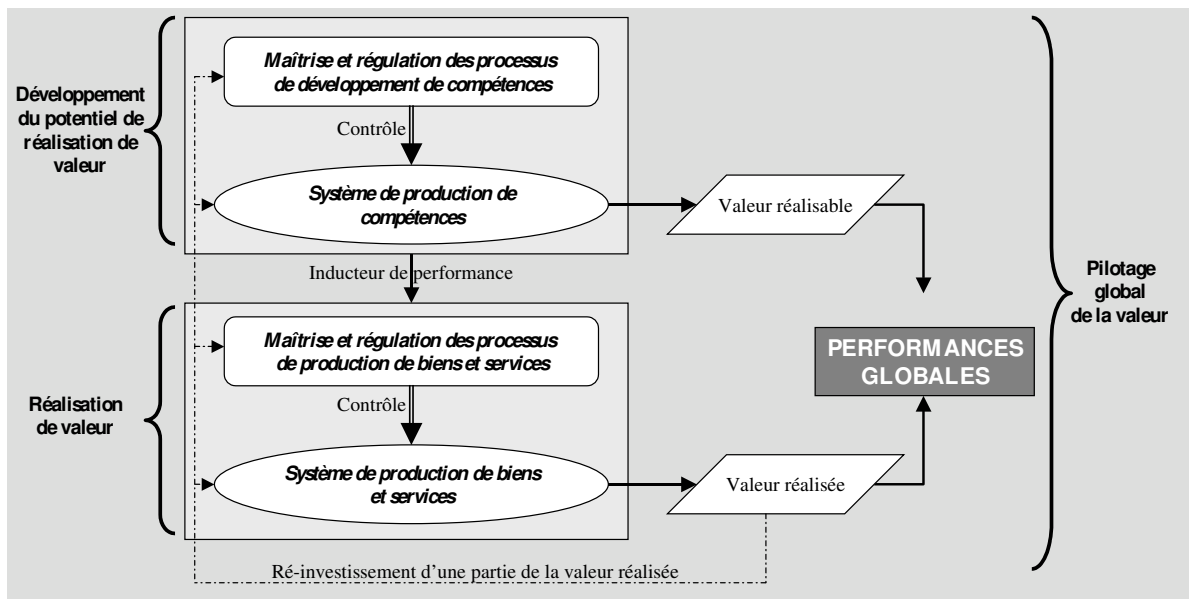

Figure 2 : La performance selon deux points de vue complémentaires

Nous proposerons donc dans la suite une méthode de conception d'un système d'indicateurs de performances pour piloter le développement des compétences d'une firme. Contrairement aux avancées actuelles dans ce domaine [KAP 98] [SVE 97], nous proposons de nous référer à un modèle formel de la compétence. Par ailleurs nous nous situons dans une perspective d'apprentissage organisationnel qui se distingue des approches patrimoniales du « capital intellectuel » [SVE 97].

\subsection{Concept de compétence}

Pour construire une approche pertinente de la performance d'un système de production de compétences, il nous semble nécessaire de préciser formellement ce que nous entendons par compétence. Un certain nombre de travaux, référencés au sein de plusieurs communautés scientifiques différentes, se sont d'ores et déjà attachés à décrire ou même à définir la notion de compétence, en se référant bien sûr aux apports des sciences humaines et sociales. La compétence est en effet avant tout une notion pluridisciplinaire abordée selon différents points de vue, desquels on peut cependant extraire plusieurs invariants. Au delà de la notion de compétence individuelle précisée ci-après, nous proposons également de définir les deux notions complémentaires de compétence collective liée à un groupe d'acteurs, puis de macrocompétence directement liée à un modèle macroscopique de l'entreprise.

Ainsi en nous référant à des travaux de sociologie comme ceux de [TER 96], [LEV 96] ou [ZAR 01], à des travaux d'économie industrielle développés par exemple par [JAC 93] ou encore à des recherches en management des organisations [BOY 82], [SAN 96], [QUE 00], nous considérons la compétence comme une notion globale recouvrant à la fois des ressources de différents types et la manière de les organiser 
pour un objectif et dans un contexte donnés. Nous nous référerons à la synthèse proposée par [BOU 99], en nous appuyant sur la définition suivante, qui s'applique directement aux compétences individuelles :

«Une compétence est une aptitude sans cesse reconstruite d'un acteur, à mobiliser de manière efficiente un certain nombre de ressources immatérielles qu'il a intériorisées (connaissances, aptitudes psychologiques et sociales,...) et de ressources matérielles de son environnement (outils, instruments, sources d'informations, etc...), pour répondre aux objectifs et au contexte propres à une situation professionnelle ».

La notion de compétence collective est restée encore peu traitée dans le domaine scientifique et tout particulièrement en génie industriel. Elle renvoie à la mobilisation d'un ensemble de compétences individuelles, coordonnées de manière efficiente dans le contexte d'une situation professionnelle. La compétence collective ne se réduit pas à la somme des compétences individuelles mais intègre également l'idée d'interactions, de réseaux, de flux d'informations propres au collectif de travail. Au delà de la recherche d'une adéquation entre compétences acquises et requises, gérer la compétence collective vise surtout à maîtriser les inducteurs de performances qui permettront une réelle efficience collective, c'est à dire l'ensemble des leviers d'action techniques, cognitifs ou sociologiques qui interviennent dans la mise en cohérence et la coordination des différentes compétences mobilisées [NON 95], [AMH 00], [воT 00], [SON 00]. Nous retenons la définition de C. H. Amherdt et F. Rabasse [AMH 00] qui caractérisent les compétences collectives comme :

« l'ensemble des savoir agir (hard/soft skills and competences) qui émergent d'une équipe de travail, combinant des ressources endogènes et exogènes de chacun des membres, et créant des compétences nouvelles issues de combinaisons synergiques de ressources ».

Enfin, nous utilisons le concept de «Macro-compétence»pour représenter la compétence à un niveau macroscopique permettant de modéliser une organisation dans sa globalité, et d'anticiper l'évolution de ses champs de compétences.

«Une macro-compétence est une agrégation de compétences collectives et individuelles qui permet de décrire de manière macroscopique le potentiel interne de compétences dont dispose une entreprise pour réaliser l'ensemble des activités nécessaires à sa production de biens et services ».

Les macro-compétences sont donc construites par regroupement de compétences individuelles ou collectives ${ }^{2}$. Elles permettront de décrire le métier propre à une entreprise, et pourront traduire son positionnement stratégique. Cependant il convient également de distinguer la notion de macro-compétences de la notion plus classique de compétence stratégique (utilisée en entreprise), qui s'appuie quant à elle

${ }^{2}$ Ce n'est donc pas la nature de l'acteur qui différencie la notion de macro-compétence de la notion de compétence collective, puisqu'une macro-compétence pourrait par exemple se référer uniquement à des acteurs individuels. 
sur un critère de jugement de valeur : ainsi une macro-compétence pourra tout à fait être considérée comme non stratégique.

\subsection{Modèle qualitatif du concept de compétence}

Les approches classiques rappelées au paragraphe 2 ont en commun de se référer à la relation \{performance - ressource\} pour décliner les notions clé de l'analyse des performances telles que l'efficacité, l'efficience et la pertinence. La mise à disposition des ressources adéquates (pertinence des ressources) et leur utilisation de manière appropriée (efficience des ressources) sont à l'origine de l'efficacité d'une entreprise mesurée par un écart entre objectifs et résultats. En ce qui concerne l'approche proposée dans cet article, la figure 2 (paragraphe 3.1) met en évidence le fait que la performance d'une organisation trouve son origine non seulement dans ses ressources (au sens matériel du terme) mais aussi dans un potentiel de valeur de nature plus complexe. Nous proposons donc d'utiliser des concepts et des modèles liés à la compétence afin de décrire ce potentiel de valeur et afin de piloter son évolution. En ce sens, c'est ici à partir de la relation \{performance - compétence\} que vont être déclinées les notions d'efficacité, d'efficience et de pertinence.

Pour modéliser l'impact de cette notion de compétence, nous proposons (figures 3.a et 3.b) le modèle qualitatif s-a-r-C (situation-acteur-ressource-Compétence) qui spécifie le concept de compétence en référence à la définition proposée au paragraphe 3.2. Ce modèle indique que la compétence émerge de l'interaction entre trois composantes essentielles : les situations, les acteurs et les ressources.

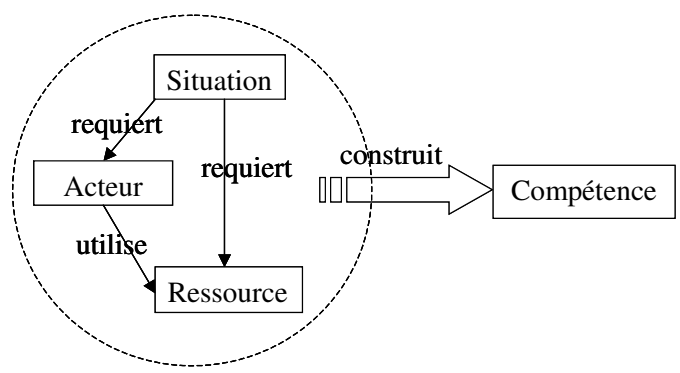

Figure 3.a : Modèle s-a-r-C

Situation $^{3}$ : En référence aux travaux de P. Zarifian [ZAR 01], le concept de « situation professionnelle » est utilisé pour représenter des tâches à réaliser ou des problèmes à résoudre, ainsi que le contexte professionnel dans lequel ils

\footnotetext{
${ }^{3}$ Dans le modèle s-a-r-C, la relation entre une situation professionnelle et une compétence est univoque : à une situation professionnelle correspond une et une seule compétence. D'autres modèles relient la compétence à niveau plus détaillé (par exemple au niveau de la tâche prescrite dans le modèle Tcaa [JiA 98]). Ce parti pris signifie que la compétence du modèle s-a-r-C est d'un niveau plus agrégé que dans d'autres modèles.
} 
apparaissent. Dans le cadre du modèle s-a-r-C, une « situation professionnelle » sera modélisée par les attributs suivants :

- un ensemble de «problèmes caractéristiques » auxquels l'acteur est confronté ;

- un « objectif» qui spécifie l'enjeu de la situation (ce qui lui donne un sens pour l'acteur) et le résultat à atteindre (variable observable qui permet de contrôler l'atteinte des objectifs);

- un « contexte ${ }^{4}$ c'est à dire un ensemble des facteurs (contrôlables) qui ont un impact sur la compétence ;

Acteur: Les acteurs désignent les ressources humaines de l'entreprise, qu'elles soient individuelles ou collectives (l'acteur intègre la notion de ressource immatérielle).

Ressource: Nous utilisons le concept de ressource pour décrire de manière exclusive les ressources de type matériel.

L'évaluation du triptyque $\{\mathrm{s}-\mathrm{a}-\mathrm{r}\}$ va fournir une première estimation ${ }^{5} \mathrm{du}$ « niveau de compétence » d'une organisation et donc du potentiel de valeur dont elle dispose : en agissant de manière convergente sur ces trois pôles de la compétence ainsi que sur leurs interactions, nous serons en mesure d'une part de développer la compétence (augmentation du potentiel de valeur disponible) et d'autre part d'augmenter la création de valeur par une meilleure utilisation de ce potentiel ${ }^{6}$.

$\mathrm{Au}$ plan conceptuel le concept de situation permet une séparation claire entre la description structurelle de la compétence, et sa description dynamique basée sur le lien entre compétence et activité (une activité mobilise des compétences). Au plan de la gestion de l'organisation, il met en exergue que l'évolution des compétences d'une entreprise suit une dynamique distincte de l'évolution de ses activités et processus. Un champ d'activité d'une entreprise pourra disparaitre sans pour autant que toutes les compétences mobilisées ne disparaissent, car les situations professionnelles demeurent dans le cadre d'autres activités.

Pour les besoins de la formalisation de ce modèle, nous avons différencié dans la figure 3.b les entités «compétence type » et «compétence instanciée ». Une « compétence type » met en relation une situation avec un type d'acteur et un type de ressource ; par exemple la compétence type «aptitude à l'élaboration de graphisme » met en relation une situation " réalisation de projets de graphisme », avec un acteur de type « graphiste », et une ressource de type « environnement d'infographie ». Cette compétence type pourra être instanciée plusieurs fois dans la même entreprise selon les graphistes effectivement au travail (Pierre, Paul..) et les environnements

\footnotetext{
${ }^{4}$ Le contexte pourra décrire par exemple des conditions de travail ou des contraintes subies par l'acteur (charge de travail, etc...) indépendantes des «problèmes caractéristiques » définissant la situation, mais qui ont un impact sur la prise en charge de la situation par l'acteur.

${ }^{5}$ Nous préciserons ultérieurement que cette première estimation dénommée «niveau de compétence théorique » devra être complétée par une seconde évaluation dénommée «niveau de compétence mesuré ».

${ }^{6}$ Notons que ce modèle met également en évidence que la compétence ne peut pas être «détenue » stricto sensu par un acteur, puisqu'elle reste dépendante des ressources matérielles qu'il peut ou non utiliser, et de la situation professionnelle portée par l'organisation.
} 
d'infographie utilisés. Ainsi l'évaluation des situations, des acteurs et des ressources fournira une estimation du niveau ${ }^{7}$ d'une « compétence instanciée ». Le niveau d'une « compétence type » s'en déduira par agrégation. Nous préciserons ces mécanismes d'évaluation au paragraphe 5.2.

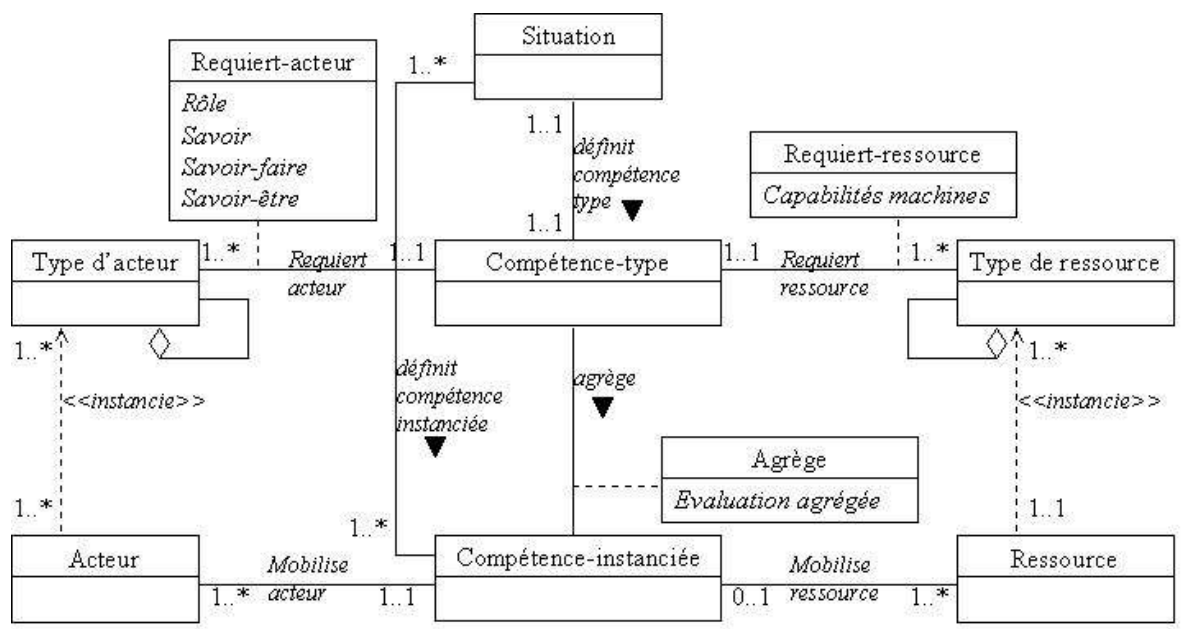

Figure 3.b : Modèle formel s-a-r-C (formalisme UML)

Enfin, soulignons que le modèle s-a-r-C met l'accent à la fois sur les aspects cognitifs et conatifs (les acteurs), technologiques (les ressources) et contextuels (les situations) de la compétence. Il permet de représenter les interactions entre ces différentes facettes de la compétence : une démarche de gestion des performances basée sur ce modèle conduit à une action coordonnée d'une part sur les trois pôles que sont les acteurs, les ressources et les situations professionnelles, et d'autre part sur les interactions entre ces pôles. Dans cette approche, la performance de l'entreprise repose sur la synergie entre ces différentes facettes de la compétence.

\section{Evaluation de l'efficacité en fonction des compétences}

Nous utiliserons ce modèle conceptuel au paragraphe 5 pour évaluer l'efficience et la pertinence des compétences, puis pour définir des actions d'amélioration des performances. Auparavant nous introduisons ci-dessous un modèle complémentaire nécessaire pour décrire les macro-compétences d'une organisation et pour établir le lien avec les compétences individuelles et collectives portées par les acteurs.

L'objectif de ce modèle présenté ci-après est notamment d'exprimer formellement la stratégie de l'entreprise pour le développement de ses compétences internes, et de

${ }^{7}$ Le « niveau » désigne le « niveau de compétence théorique » définit ci-avant. 
quantifier cette stratégie par les priorités de développement qu'elle se fixe. Nous proposons de modéliser ces priorités stratégiques en utilisant la théorie des sousensembles flous [ZAD 65] qui nous fournit une algèbre adéquate pour le traitement de valeurs qualitatives.

L'identification des ensembles de compétences et de macro-compétences mobilisées par une entreprise suppose de s'appuyer sur des dictionnaires ou référentiels de compétences. Dans l'état actuel de nos travaux de recherche nous nous référons au dictionnaire de compétences proposé par le ROME (Répertoire Opérationnel des Métiers et de l'Emploi). Le ROME présente en effet l'avantage de fournir un standard à l'échelle nationale, de décrire les compétences de manière agrégée, et de se prêter à la définition d'ensembles de compétences au contraire d'autres classifications telles que le code $\mathrm{NAF}^{8}$.

\subsection{Mesure du niveau et des objectifs de compétences}

Soit $C=\left\{C_{1}, C_{2}, \ldots C_{h}\right\}$ un ensemble fini d'ordre h de compétences-types ${ }^{9}$. C est défini à partir d'un référentiel ou dictionnaire de compétences du type ROME. Soit $\mathrm{C}_{\mathrm{i}}$ un élément de $\mathrm{C}$. On note $\tilde{X}$ le sous-ensemble flou de $\mathrm{C}$ défini par :

$$
\tilde{X}=\left\{C_{1}\left|\mu_{X}\left(C_{1}\right), C_{2}\right| \mu_{X}\left(C_{2}\right), \ldots, C_{h} \mid \mu_{X}\left(C_{h}\right)\right\}
$$

où $\mu_{\mathrm{X}}\left(\mathrm{C}_{\mathrm{i}}\right)$ est la fonction caractéristique d'appartenance de $\mathrm{C}_{\mathrm{i}}$ dans $\tilde{X}, \mu_{\mathrm{X}}\left(\mathrm{C}_{\mathrm{i}}\right)$ prenant ses valeurs dans $[0,1]$.

La fonction caractéristique est l'évaluation du degré d'appartenance d'une compétence au sous-ensemble flou $\tilde{X}$. Si $\mu_{\mathrm{X}}\left(\mathrm{C}_{\mathrm{i}}\right)=1$, la compétence $\mathrm{C}_{\mathrm{i}}$ appartient totalement à $\tilde{X}$. Si $\mu_{\mathrm{X}}\left(\mathrm{C}_{\mathrm{i}}\right)=0$, la compétence $\mathrm{C}_{\mathrm{i}}$ n'appartient pas à $\tilde{X}$. Entre ces deux extrêmes, une compétence peut appartenir faiblement (par exemple $\mu_{X}\left(C_{i}\right)=$ $0.2)$ ou fortement $\left(\mu_{\mathrm{X}}\left(\mathrm{C}_{\mathrm{i}}\right)=0.8\right)$ à $\tilde{X}$.

Nous noterons $\mathrm{C}(\mathrm{E})$ le sous-ensemble net (i. e. non flou) de $\mathrm{C}$ d'ordre $\mathrm{n}(n \leq h)$ des compétences à évaluer dans une entreprise E. C(E) contient les compétences présentes dans l'entreprise ainsi que les compétences qu'elle souhaite acquérir. Nous noterons $\tilde{R}(\mathrm{E})$ le sous-ensemble flou de $\mathrm{C}(\mathrm{E})$ qui décrit le niveau de compétence de l'entreprise $\mathrm{E}$ mesuré sur les éléments de $\mathrm{C}(\mathrm{E}) . \tilde{R}(\mathrm{E})$ est le résultat d'une mesure ${ }^{10}$, et constitue une évaluation du noyau de compétences (core competencies) de

\footnotetext{
${ }^{8}$ Les codes de type NAF permettent uniquement une classification des entreprises par domaine d'activité de manière exclusive ; le ROME permet de définir une fonction d'appartenance non exclusive du type « une entreprise possède telle ou telle compétence ».

${ }^{9}$ Selon la définition donnée dans le modèle s-a-r-C, et que nous appellerons simplement « compétence » par la suite, à la différence des « compétences instanciées ».

${ }^{10}$ La définition des modalités opérationnelles de mesure des compétences sort du champ de cet article. Le lecteur pourra se reporter à ce sujet aux travaux de [BOT 00], chapitre 4 «L'évaluation des compétences ».
} 
l'entreprise E. Par exemple, $\mu_{R(E)}\left(C_{i}\right)=0.2$ signifie qu'il a été évalué que la compétence $\mathrm{C}_{\mathrm{i}}$ est faiblement maîtrisée par l'entreprise $\mathrm{E}$.

Nous noterons $\tilde{O}(\mathrm{E})$ le sous-ensemble flou de $\mathrm{C}(\mathrm{E})$ qui décrit l'objectif d'acquisition et de maîtrise d'un ensemble de compétences par une entreprise $\mathrm{E}$ : $\mu_{\mathrm{O}(\mathrm{E})}\left(\mathrm{C}_{\mathrm{i}}\right)=0.8$ signifie que l'entreprise a pour objectif de maîtriser fortement la compétence $\mathrm{C}_{\mathrm{i}}$.

\subsection{Evaluation de l'efficacité du processus de pilotage des compétences}

Afin de mesurer l'efficacité du processus de pilotage des compétences de l'entreprise E, nous devons calculer l'écart entre le résultat et l'objectif ${ }^{11}$. Pour cela, nous pouvons calculer la distance de Hamming ${ }^{12}$ généralisée relative $\mathrm{d}_{1}(\widetilde{R}(\mathrm{E})$, $\tilde{O}(\mathrm{E}))$ entre les deux sous-ensembles flous $\widetilde{R}(\mathrm{E})$ et $\tilde{O}(\mathrm{E})$ :

$$
d_{1}(\tilde{R}(E), \tilde{O}(E))=\frac{1}{n} \sum_{i=1}^{n}\left|\mu_{R(E)}\left(C_{i}\right)-\mu_{O(E)}\left(C_{i}\right)\right|
$$

Si l'on souhaite différencier la contribution des différentes compétences à la mesure de l'efficacité, il est possible d'utiliser une mesure pondérée :

$$
d_{2}(\tilde{R}(E), \tilde{O}(E))=\sum_{i=1}^{n} p_{i}\left|\mu_{R(E)}\left(C_{i}\right)-\mu_{O(E)}\left(C_{i}\right)\right| \text { avec } p_{i} \geq 0, \text { et } \sum_{i=1}^{n} p_{i}=1
$$

L'efficacité $\varepsilon_{1}$ ou $\varepsilon_{2}$ est ensuite obtenue en prenant le complément à 1 de la distance :

$$
\begin{aligned}
& \varepsilon_{1}=1-d_{1}(\tilde{R}(E), \tilde{O}(E)) \\
& \varepsilon_{2}=1-d_{2}(\tilde{R}(E), \tilde{O}(E))
\end{aligned}
$$

D'autre part, les mesures de distance $\mathrm{d}_{1}$ et $\mathrm{d}_{2}$ entre les sous-ensembles flous $\tilde{R}(\mathrm{E})$ et $\tilde{O}(\mathrm{E})$, évaluent les écarts dans les deux sens. Elles mesurent autant un déficit de compétence, qu'une «sur-compétence » par rapport à l'objectif. Si l'on ne souhaite pas intégrer une composante de sur-qualité dans la mesure de l'efficacité, il faut extraire du calcul les écarts provenant des sur-compétences.

Dans ce but, on isole dans $\tilde{R}(\mathrm{E})$ les niveaux de compétences uniquement requis pour l'objectif en réalisant l'intersection au sens des sous-ensembles flous entre

\footnotetext{
${ }^{11}$ Selon la définition de l'efficacité donnée au paragraphe 2.

12 La distance de Hamming correspond au cas particulier où $\alpha=1$ dans la distance $\sum\left(\left|x^{\alpha}-y^{\alpha}\right|\right)^{1 / \alpha}$ avec $\alpha \geq 1$. Une perspective est d'étudier l'usage d'autres distances.
} 
$\tilde{R}(\mathrm{E})$ et $\tilde{O}(\mathrm{E})$. La fonction caractéristique d'appartenance de $\mathrm{C}_{\mathrm{i}}$ dans $\tilde{R}(E) \cap \widetilde{O}(E)$ est définie par :

$$
\mu_{R(E) \cap O(E)}\left(C_{i}\right)=\operatorname{Min}\left(\mu_{R(E)}\left(C_{i}\right), \mu_{O(E)}\left(C_{i}\right)\right), \forall C_{i} \in \mathrm{C}(\mathrm{E})
$$

On calcule alors la nouvelle efficacité notée $\varepsilon_{1}^{\prime}$ ou $\varepsilon_{2}^{\prime}$ par les distances $\mathrm{d}_{1}$ ou $\mathrm{d}_{2}$ :

$$
\begin{aligned}
& d_{1}(\tilde{R}(E) \cap \tilde{O}(E), \tilde{O}(E))=\frac{1}{n} \sum_{i=1}^{n}\left|\mu_{R(E) \cap O(E)}\left(C_{i}\right)-\mu_{O(E)}\left(C_{i}\right)\right| \\
& d_{2}(\tilde{R}(E) \cap \tilde{O}(E), \tilde{O}(E))=\sum_{i=1}^{n} p_{i}\left|\mu_{R(E) \cap O(E)}\left(C_{i}\right)-\mu_{O(E)}\left(C_{i}\right)\right| \\
& \text { puis }: \varepsilon_{1}^{\prime}=1-d_{1}(\tilde{R}(E) \cap \tilde{O}(E), \tilde{O}(E)) \\
& \text { et } \varepsilon_{2}^{\prime}=1-d_{2}(\tilde{R}(E) \cap \tilde{O}(E), \tilde{O}(E))
\end{aligned}
$$

\subsection{Mesure de l'efficacité en fonction des macro-compétences}

La stratégie d'une entreprise est généralement exprimée non pas en terme de compétences détaillées mais plutôt par des objectifs plus agrégés, que nous avons dénommés macro-compétences. Il est donc utile de pouvoir calculer une efficacité par rapport à des objectifs de macro-compétences. Pour cela, nous supposons que l'objectif précédent $\tilde{O}(\mathrm{E})$ est décomposé par l'entreprise en $m$ objectifs de macrocompétences $\tilde{M}_{j}(\mathrm{E})$.

Pour que la politique de l'entreprise soit cohérente, on impose que $\tilde{M}_{j} \subset \tilde{O}, \forall j$ entre 1 et $\mathrm{m}$, aux sens des sous-ensembles flous, c'est à dire pour tout objectif de macro-compétence $\tilde{M}_{j}(\mathrm{E})$ et pour toute compétence $\mathrm{C}_{\mathrm{i}}$ :

$$
\mu_{M_{j}(E)}\left(C_{i}\right) \leq \mu_{O(E)}\left(C_{i}\right)
$$

Cela signifie qu'aucun objectif de macro-compétence ne dépasse l'objectif global. Si l'on souhaite que la totalité de l'objectif global soit exprimée dans la somme des objectifs de macro-compétences, alors on impose $\bigcup_{j}\left(\tilde{M}_{j}\right)=\tilde{O}$, c'est à dire que pour toute compétence $\mathrm{C}_{\mathrm{i}}: \operatorname{Max}_{j}\left(\mu_{M_{j}(E)}\left(C_{i}\right)\right)=\mu_{O(E)}\left(C_{i}\right)$

De plus, on accepte qu'il existe des macro-compétences $\tilde{M}_{j}(\mathrm{E})$ et $\tilde{M}_{k}(\mathrm{E})$ d'intersection $\tilde{M}_{j}(E) \cap \tilde{M}_{k}(E)$ non vide, c'est à dire qu'il peut exister des 
compétences $C_{\mathrm{i}}$ pour lesquelles $\operatorname{Min}\left(\mu_{M_{j}(E)}\left(C_{i}\right), \mu_{M_{k}(E)}\left(C_{i}\right)\right) \neq 0$, pour prendre en compte le cas de la contribution d'une même compétence $\mathrm{C}_{\mathrm{i}}$ à plusieurs macrocompétences .

Il est alors possible de mesurer l'efficacité du système de gestion des macrocompétences d'une entreprise. Pour cela, nous déterminons tout d'abord le sousensemble flou $\tilde{R}_{j}(\mathrm{E})$ défini par $\tilde{R}_{j}(E)=\tilde{R}(E) \cap \tilde{M}_{j}(E)$ qui exprime la contribution des compétences de l'entreprise à l'atteinte de l'objectif de macrocompétences $\tilde{M}_{j}(\mathrm{E})$. La composante de sur-qualité est automatiquement supprimée ici par l'opération précédente d'intersection. On détermine ensuite dans $\tilde{R}_{j}(\mathrm{E})$ le nombre $\mathrm{n}_{\mathrm{j}}$ de compétences $\mathrm{C}_{\mathrm{i}}$ telle que $\mu_{R_{j}(E)}\left(C_{i}\right) \neq 0$, de façon à diviser l'écart par le nombre de compétences effectivement visées dans le cadre de la macrocompétence.

On mesure ensuite l'écart entre ce résultat et l'objectif par la distance de Hamming généralisée non relative $\mathrm{d}_{3}$ :

$$
\begin{aligned}
& d_{3}\left(\tilde{R}_{j}(E), \tilde{M}_{j}(E)\right)=\sum_{i=1}^{n}\left|\mu_{R_{j}(E)}\left(C_{i}\right)-\mu_{M_{j}(E)}\left(C_{i}\right)\right| \\
& \text { Puis on calcule l'efficacité par : } \varepsilon_{3, j}^{\prime}=1-\frac{d_{3}\left(\tilde{R}_{j}(E), \tilde{M}_{j}(E)\right)}{n_{j}}
\end{aligned}
$$

\section{Evaluation de la pertinence et de l'efficience}

\subsection{Caractérisation des moyens}

Comme cela a été précisé au paragraphe 2, la pertinence correspond à l'écart entre l'objectif et les moyens mis en œuvre pour l'atteindre. Quant à l'efficience, elle correspond à l'écart entre les résultats et ces mêmes moyens. Pour évaluer la pertinence et l'efficience du processus de développement des compétences, il convient à présent de caractériser les moyens mis en œuvre. Conformément au modèle s-a-r-C, nous allons modéliser ces moyens par les concepts de situation, d'acteur et de ressource. Nous les caractériserons par des indicateurs qui nous permettront d'évaluer le niveau de compétence théorique déjà introduit au paragraphe 3.3. Nous allons également utiliser la théorie des sous-ensembles flous pour représenter qualitativement ces trois composantes.

Nous définissons donc :

- $S=\left\{S_{1}, S_{2}, \ldots S_{p}\right\}$ un ensemble fini d'ordre $p$ de situations, caractérisées par l'indicateur «degré de maitrise » de l'entreprise sur la situation professionnelle ${ }^{13}$.

${ }^{13}$ Pour évaluer le «degré de maîtrise » différents facteurs pourront être pris en compte, comme la fréquence avec laquelle l'entreprise est confrontée à cette situation, mais également le fait que la situation 
Rappelons qu'à chaque $S_{i}$ correspond une compétence $C_{i}$ et une seule.

- $A=\left\{A_{1}, A_{2}, \ldots A_{q}\right\}$ un ensemble fini d'ordre q d'acteurs, caractérisés par l'indicateur «adéquation de l'acteur » par rapport à sa disponibilité et aux capacités cognitives requises par la situation.

- $Q=\left\{Q_{1}, Q_{2}, \ldots Q_{r}\right\}$ un ensemble fini d'ordre $r$ de ressources, caractérisées par l'indicateur «niveau de service» par rapport à la disponibilité et aux capabilités technologiques requises par la situation.

Comme pour l'ensemble $\mathrm{C}$ de compétences, ces trois ensembles $\mathrm{S}$, A et $\mathrm{Q}$ constituent des référentiels de situations, de ressources et d'acteurs. L'ensemble $\mathrm{S}$ peut être construit à partir de descriptifs de situations issus de référentiels tels que le ROME, l'ensemble A depuis la liste des postes (ou emplois) habituels d'un secteur industriel donné, et l'ensemble Q à partir de la liste des ressources technologiques habituelles de ce secteur. Nous donnerons au paragraphe 6 des exemples de bases pour ces référentiels.

Nous proposons trois indicateurs relativement génériques pour les situations, les acteurs et les ressources. Ils pourront être spécifiés plus précisément pour s'adapter au contexte de chaque entreprise, sans pour autant modifier les modalités mathématiques de calculs du niveau de compétence, de la pertinence ou de l'efficience précisés ci-après.

Nous noterons $\tilde{S}$ (E) (respectivement $\tilde{A}(\mathrm{E})$ et $\tilde{Q}(\mathrm{E})$ ) le sous-ensemble flou de $\mathrm{S}$ (resp. de A et de Q) qui décrit le degré de maîtrise des situations professionnelles par l'entreprise E (resp. l'adéquation des acteurs, ainsi que le niveau de service des ressources de E).

Ainsi, pour le sous-ensemble flou $\tilde{S}(\mathrm{E})$, lorsque $\mu_{\mathrm{S}(\mathrm{E})}\left(\mathrm{S}_{\mathrm{i}}\right)=1$, l'entreprise maîtrise complètement la situation professionnelle $S_{\mathrm{i}}$. Par contre $\mu_{\mathrm{S}(\mathrm{E})}\left(\mathrm{S}_{\mathrm{i}}\right)=0,1$ traduit une situation $S_{i}$ faiblement maîtrisée par l'entreprise, par exemple parce que cette situation professionnelle intervient rarement dans son activité ou parce qu'elle n'a pas été rationalisée. Les valeurs de $\mu_{\mathrm{S}(\mathrm{E})}, \mu_{\mathrm{A}(\mathrm{E})}$ et $\mu_{\mathrm{Q}(\mathrm{E})}$ sont évaluées par audit et interview dans l'entreprise.

\subsection{Construction d'un niveau de compétence théorique}

Nous déterminons à présent le niveau théorique (ou encore standard) de compétences que l'entreprise $\mathrm{E}$ devrait obtenir à partir des moyens qu'elle a mis en œuvre. Ce niveau noté $\tilde{N}$ (E) est un sous-ensemble flou de l'ensemble $\mathrm{C}(\mathrm{E})=\left\{\mathrm{C}_{1}\right.$, $\left.\mathrm{C}_{2}, \ldots \mathrm{C}_{\mathrm{n}}\right\}$ des compétences de l'entreprise E. Pour caractériser ce sous-ensemble flou $\tilde{N}(\mathrm{E})$, il faut évaluer les $\mu_{\mathrm{N}(\mathrm{E})}\left(\mathrm{C}_{\mathrm{i}}\right)$ qui définissent le degré d'appartenance de chaque compétence-type $\mathrm{C}_{\mathrm{i}}$ à $\tilde{N}(\mathrm{E})$. Pour cela, nous déterminons tout d'abord un niveau de maîtrise théorique $\mu\left(\mathrm{C}_{\mathrm{i}, \mathrm{j}, \mathrm{k}}\right)$ pour les compétences instanciées à partir des

soit soumise ou non à un processus de rationalisation (analyse, explicitation, contrôle par des indicateurs de performances, ...). On notera également que les pôles «acteurs » et «ressources » sont évalués contextuellement, c'est à dire par rapport à la situation professionnelle. 
trois sous-ensembles flous $\tilde{S}(\mathrm{E}), \tilde{A}(\mathrm{E})$ et $\tilde{Q}(\mathrm{E})$, c'est à dire en raisonnant en termes de fonctions d'appartenance :

$$
\mu\left(C_{i, j, k}\right)=f\left(\mu_{S(E)}\left(S_{i}\right), \mu_{A(E)}\left(A_{j}\right), \mu_{Q(E)}\left(Q_{k}\right)\right)
$$

où :

- f est une fonction de variables floues,

- $\quad \mathrm{S}_{\mathrm{i}}$ est la situation professionnelle associée de façon univoque à la compétence $\mathrm{C}_{\mathrm{i}}$ par le modèle s-a-r-C,

- $\quad \mathrm{C}_{\mathrm{i}, \mathrm{j}, \mathrm{k}}$ est la compétence $\mathrm{C}_{\mathrm{i}}$, instanciée selon l'acteur $\mathrm{j}$ et la ressource $\mathrm{k}$,

- $\quad A_{j}$ est l'acteur porteur de la compétence instanciée $C_{i, j, k}$,

- $\quad \mathrm{Q}_{\mathrm{k}}$ est la ressource contribuant à la construction de la compétence instanciée $\mathrm{C}_{\mathrm{i}, \mathrm{j}, \mathrm{k}}$.

La fonction $\mathrm{f}$ peut être obtenue au moins de deux façons :

- Soit à partir d'enquêtes sur les meilleures pratiques d'un secteur industriel (benchmark), permettant de quantifier les quadruplets (acteurs, ressources, activités, compétences) du modèle s-a-r-C. Ainsi le quadruplet $\left(\mathrm{S}_{\mathrm{i}}, \mathrm{A}_{\mathrm{j}}, \mathrm{Q}_{\mathrm{k}}, \mathrm{C}_{\mathrm{i}, \mathrm{j}, \mathrm{k}}\right)$ prendra par exemple la valeur $(0.8,0.6,0.7,0.6)$ qui sera considérée comme une combinaison performante du secteur. De nombreuses autres combinaisons peuvent mener à la valeur $\mu\left(\mathrm{C}_{\mathrm{i}, \mathrm{j}, \mathrm{k}}\right)=0.6$, comme par exemple $(0.6,0.8,0.7,0.6)$ ce qui correspond à une situation pour laquelle l'effort de rationalisation est moindre mais le niveau de l'acteur est plus élevé.

- Soit en utilisant la logique flou pour calculer le niveau de compétence résultant de la combinaison logique de la situation, de l'acteur et de la ressource :

$\mu\left(\mathrm{C}_{\mathrm{i}, \mathrm{j}, \mathrm{k}}\right)=\mu_{\mathrm{S}(\mathrm{E})}\left(\mathrm{S}_{\mathrm{i}}\right)$ et $\mu_{\mathrm{A}(\mathrm{E})}\left(\mathrm{A}_{\mathrm{j}}\right)$ et $\mu_{\mathrm{Q}(\mathrm{E})}\left(\mathrm{Q}_{\mathrm{k}}\right)$ en utilisant l'opérateur « et » de la logique flou, c'est à dire :

$$
\mu\left(C_{i, j, k}\right)=\operatorname{Min}\left(\mu_{S(E)}\left(S_{i}\right), \mu_{A(E)}\left(A_{j}\right), \mu_{Q(E)}\left(Q_{k}\right)\right)
$$

Cette seconde modalité pour définir la fonction $\mathrm{f}$ est naturellement réductrice mais possède l'avantage de la simplicité opératoire. Nous l'utiliserons dans l'exemple industriel développé au paragraphe 6 .

Dans les deux cas, et une fois la fonction $\mathrm{f}$ construite, il reste à calculer le degré d'appartenance de chaque compétence-type $\mathrm{C}_{\mathrm{i}}$ à $\tilde{N}(\mathrm{E})$. Les valeurs $\mu_{\mathrm{N}(\mathrm{E})}\left(\mathrm{C}_{\mathrm{i}}\right)$ sont calculées par une fonction d'agrégation notée g à partir des valeurs $\mu\left(\mathrm{C}_{\mathrm{i}, \mathrm{j}, \mathrm{k}}\right)$ :

$$
\mu_{\mathrm{N}(\mathrm{E})}\left(\mathrm{C}_{\mathrm{i}}\right)=\mathrm{g}\left(\mu\left(\mathrm{C}_{\mathrm{i}, \mathrm{j}, \mathrm{k}}\right)\right)
$$

La fonction d'agrégation la plus simple sera une moyenne des $\mu\left(\mathrm{C}_{\mathrm{i}, \mathrm{j}, \mathrm{k}}\right)$ sur les acteurs $\mathrm{A}_{\mathrm{j}}$ et sur les ressources $\mathrm{Q}_{\mathrm{k}}$. A l'arrivée, nous obtenons ainsi une estimation $\mathrm{du}$ sous-ensemble flou $\tilde{N}$ (E) représentant le niveau théorique (ou standard) de compétences que les meilleures entreprises du secteur obtiendraient à partir des 
niveaux de situations professionnelles, d'acteurs et de ressources équivalents à ceux évalués dans $\tilde{S}(\mathrm{E}), \tilde{A}(\mathrm{E})$ et $\tilde{Q}(\mathrm{E})$.

\subsection{Evaluation de la pertinence}

Il devient à présent possible de mesurer la pertinence, notée ici $\rho$, par l'écart entre l'objectif visé $\tilde{O}(\mathrm{E})$ et le niveau de compétence théorique $\tilde{N}(\mathrm{E})$. Cet écart est calculé comme précédemment par une distance de Hamming généralisée relative $d_{1}\left(\right.$ ou $d_{2}$ si l'on souhaite pondérer l'importance des compétences $\mathrm{C}_{\mathrm{i}}$ ) :

$$
\begin{aligned}
& d_{1}(\tilde{N}(E), \tilde{O}(E))=\frac{1}{n} \sum_{i=1}^{n}\left|\mu_{N(E)}\left(C_{i}\right)-\mu_{O(E)}\left(C_{i}\right)\right| \\
& \rho_{1}=1-d_{1}(\tilde{N}(E), \tilde{O}(E))
\end{aligned}
$$

Il est également possible de détecter l'excédent de moyens mis en ouvre en calculant $\mathrm{d}_{1}(\tilde{N}(E) \cap \tilde{O}(E), \tilde{O}(E))$, puis $\rho_{1}^{\prime}=1-d_{1}(\tilde{N}(E) \cap \tilde{O}(E), \tilde{O}(E))$

L'excédent de moyens mis en œuvre est obtenu par $\rho_{1}^{\prime}-\rho_{1}$.

Pour calculer la pertinence vis-à-vis de l'objectif $\tilde{M}_{j}(\mathrm{E})$ de macro-compétences $\mathrm{j}$, nous déterminons tout d'abord le sous-ensemble flou $\tilde{N}_{j}(\mathrm{E})$ défini par $\tilde{N}_{j}(E)=\tilde{N}(E) \cap \tilde{M}_{j}(E)$ qui isole parmi les moyens de l'entreprise ceux qui contribuent à l'atteinte de l'objectif de macro-compétences $\tilde{M}_{j}($ E). Comme pour $\tilde{R}_{j}$ (E) la composante de sur-qualité est automatiquement supprimée ici par l'opération d'intersection. On détermine ensuite dans $\tilde{N}_{j}\left(\right.$ E) le nombre $\mathrm{n}_{\mathrm{j}}$ de compétences $C_{\mathrm{i}}$ telle que $\mu_{N_{j}(E)}\left(C_{i}\right) \neq 0$, pour diviser l'écart par le nombre de compétences visées par la macro-compétence.

On mesure ensuite comme dans le cas de la formule [12] l'écart entre ce résultat et l'objectif par la distance de Hamming généralisée non relative $\mathrm{d}_{3}$ :

$$
d_{3}\left(\tilde{N}_{j}(E), \tilde{M}_{j}(E)\right)=\sum_{i=1}^{n}\left|\mu_{N_{j}(E)}\left(C_{i}\right)-\mu_{M_{j}(E)}\left(C_{i}\right)\right|
$$

Puis on calcule la pertinence des moyens vis-à-vis d'une macro-compétence par :

$$
\rho_{3, j}^{\prime}=1-\frac{d_{3}\left(\tilde{N}_{j}(E), \tilde{M}_{j}(E)\right)}{n_{j}}
$$


En revanche, il faut être plus prudent sur l'interprétation de la mesure directe $\mathrm{d}_{1}\left(\tilde{N}(E), \tilde{M}_{j}(E)\right)$ car des moyens peuvent paraître sur-dimensionnés au titre de la macro-compétence $\tilde{M}_{j}(\mathrm{E})$, mais nécessaires pour d'autres macro-compétences.

L'intérêt de cette approche est également d'induire une synergie sur les actions concrètes à mener dans l'entreprise en vue d'améliorer la pertinence des compétences déployées. Ainsi, en cas d'identification d'un manque de pertinence, les leviers d'action possibles peuvent intervenir de manière complémentaire sur un ou plusieurs des pôles «situation», «acteur», « ressource» (tableau 1). Ce pilotage coordonné sur les trois pôles de la compétence évite par exemple que l'effet bénéfique d'un investissement technologique soit amoindri par un manque de formation des acteurs ou par un manque de rationalisation de la situation.

\begin{tabular}{|l|l|}
\hline \multicolumn{1}{|c|}{ Composante de la compétence } & \multicolumn{1}{c|}{ Exemples de leviers d'action } \\
\hline Situation & $\begin{array}{l}\text { Analyse et rationalisation de la situation } \\
\text { Contrôle de la situation par indicateurs de performances } \\
\text { Formation des acteurs à la maîtrise de cette situation }\end{array}$ \\
\hline Acteur & $\begin{array}{l}\text { Développement des compétences des acteurs } \\
\text { Développement de la compétence collective } \\
\text { Recrutement }\end{array}$ \\
\hline Ressources & Investissement technologique \\
\hline
\end{tabular}

Tableau 1 : Leviers d'action pour la pertinence

Notons que les leviers d'action pour le développement de la compétence cités ici se rapportent uniquement à une recherche de pertinence. D'autres leviers d'action seront associés à une recherche d'efficience (paragraphe suivant).

\subsection{Evaluation de l'efficience}

Rappelons que le sous-ensemble flou $\tilde{R}(\mathrm{E})$ représente le niveau de compétence mesuré pour l'entreprise E. Pour déterminer l'efficience $\eta_{1}$, nous vérifions en préalable que $\tilde{R}(E) \subseteq \tilde{N}(E)$, c'est à dire que le niveau de compétence mesuré dans le cas de l'entreprise E reste inférieur ou égal aux meilleures pratiques du secteur. En cas contraire les pratiques de l'entreprise $\mathrm{E}$ doivent remplacer celles du référentiel $\tilde{N}(\mathrm{E})$.

Ensuite nous calculons $d_{1}(\tilde{N}(E), \tilde{R}(E))=\frac{1}{n} \sum_{i=1}^{n}\left|\mu_{N(E)}\left(C_{i}\right)-\mu_{R(E)}\left(C_{i}\right)\right|$

puis : $\eta_{1}=1-d_{1}(\tilde{N}(E), \tilde{R}(E))$ 
Au contraire de la pertinence, un manque d'efficience n'est pas lié à une faiblesse de l'une ou l'autre des trois composantes de la compétence, mais à d'autres facteurs qui interviennent sur l'émergence effective de la compétence. D'une manière générale on peut dire que ces facteurs d'efficience sont liés aux interactions entre l'acteur, la situation et les ressources. Il convient donc d'analyser précisément ces interactions pour identifier les leviers d'action potentiels. A titre d'exemple nous en soulignons quelques uns ci-dessous.

\begin{tabular}{|l|l|}
\hline \multicolumn{1}{|c|}{ Type d'interaction } & \multicolumn{1}{c|}{ Exemples de facteurs d'efficience } \\
\hline Situation-Acteur & $\begin{array}{l}\text { Motivations (facteurs conatifs) } \\
\text { Autonomie (zone d'autonomie suffisante pour la tâche ?) } \\
\text { Ergonomie cognitive de la situation } \\
\text { Fréquence des aléas humains }\end{array}$ \\
\hline Situation-Ressource & $\begin{array}{l}\text { Répartition des charges sur les ressources technologiques } \\
\text { Fréquence des aléas technologiques }\end{array}$ \\
\hline Acteur-Ressources & $\begin{array}{l}\text { Ergonomie } \\
\text { Confiance (la ressource est-elle « sûre » pour l'acteur ?) }\end{array}$ \\
\hline
\end{tabular}

Tableau 2 : Leviers d'action pour l'efficience

Au delà de ces facteurs d'efficience une analyse de causalité contextuelle et propre à chaque entreprise doit être réalisée pour identifier les leviers d'action critiques. Par exemple, un manque de disponibilité des ressources pourra être lié à une mauvaise gestion des flux autant qu'à un dimensionnement erroné ; de même un manque de motivation des acteurs pourra être lié à des facteurs conatifs aussi divers que la rémunération ou la reconnaissance personnelle.

\section{Exemple : tableau de bord pour le pilotage des compétences}

\subsection{Contexte de l'étude de cas}

L'étude de cas traitée dans cet article est extraite d'une coopération avec le groupement d'entreprises ARTIC ${ }^{14}$. ARTIC est une association d'une vingtaine d'entreprises Roannaises qui exercent leurs activités dans les domaines de l'informatique (matériels, logiciels, internet), de la bureautique, de l'automatisme et des télécommunications. Afin de mieux gérer l'émergence de coopération à

\footnotetext{
${ }^{14}$ Coopération développée dans le cadre du projet GRECOPME II financée par la région Rhône Alpes et réunissant quatre laboratoires de recherche : le département Organisation et Modélisation des Systèmes Industriels (OMSI) de l'Ecole Nationale Supérieure des Mines de Saint Etienne, le Laboratoire d'Analyse des Signaux et des Processus Industriels (LASPI) de l'Université Jean Monnet / IUT Roanne, le Centre de Recherches en Economie de l'Université de Saint-Etienne (CREUSET), le laboratoire Productique et Informatique des Systèmes Manufacturiers (PRISMa) de l'INSA de Lyon et de l'Université Claude Bernard Lyon I.
} 
l'intérieur de cette association, ARTIC s'est engagée dans un processus de cartographie et d'évaluation des macro-compétences. Cette étude a notamment pour objet de mettre en place des processus collectifs de développement des compétences.

L'objet de cet article n'étant pas de traiter de la coopération inter-entreprises, nous nous limiterons à citer l'exemple de l'une des entreprises du groupement travaillant dans le domaine de la réalisation d'applications informatiques basées sur la technologie internet. A travers cette étude de cas, nous allons illustrer la construction complète d'un tableau de bord pour le pilotage du développement de compétences en entreprise.

\subsection{Application du modèle}

\subsubsection{Formalisation des données}

Pour qualifier les compétences, nous utilisons les niveaux du référentiel ROME auxquels nous faisons correspondre des valeurs pour la fonction d'appartenance :

\begin{tabular}{|c|c|c|}
\hline Niveau & Désignation & $\begin{array}{c}\text { Valeur de fonction } \\
\text { d'appartenance }\end{array}$ \\
\hline 0 & absence & 0 \\
\hline 1 & sensibilisation & 0,25 \\
\hline 2 & capacité à mettre en pratique & 0,5 \\
\hline 3 & maîtrise & 0,75 \\
\hline 4 & expertise, possibilité de faire évoluer la fonction, formation & 1 \\
\hline
\end{tabular}

Tableau 3 : Niveaux de qualification des compétences

Pour simplifier la présentation de cette étude de cas, nous nous limiterons dans l'article à décrire les compétences techniques de l'entreprise, même si ses compétences managériales ont également fait l'objet de l'étude. Nous avons ainsi identifié dix compétences techniques de base et quatre macro-compétences ${ }^{15}$. Les objectifs sur les compétences identifiées, ainsi que les objectifs sur les macrocompétences et les résultats par compétence sont regroupés dans le tableau 4.

L'ensemble des données synthétisées dans ce tableau a été l'objet d'un audit approfondi de l'entreprise [BOU 02]. Dans notre démarche, la stratégie de développement des compétences de l'entreprise est exprimée à la fois par un objectif sur le niveau de chacune des dix compétences de base, et par un objectif sur chaque macro-compétence qui traduit la contribution d'une compétence de base à la macrocompétence. Ces objectifs sont exprimés par le dirigeant de l'entreprise.

\footnotetext{
${ }^{15}$ Les dénominations utilisées dans le tableau sont des abréviations. A titre d'exemple MC1 désigne plus précisément l'aptitude à maîtriser des processus de traitement de l'image et MC2 désigne l'aptitude à maîtriser des processus de développement de logiciels. Cette formalisation du vocabulaire est nécessaire également pour les compétences de base.
} 


\begin{tabular}{|c|c|c|c|c|c|c|c|}
\hline & & $\tilde{O}(\mathrm{E})$ & $\tilde{M}_{1}(\mathrm{E})$ & $\tilde{M}_{2}(\mathrm{E})$ & $\tilde{M}_{3}(\mathrm{E})$ & $\tilde{M}_{4}(\mathrm{E})$ & $\tilde{R}(\mathrm{E})$ \\
\hline $\begin{array}{l}\text { Compé- } \\
\text { tences }\end{array}$ & Désignation & Objectifs & $\begin{array}{l}\text { Traitement } \\
\text { d'images }\end{array}$ & \begin{tabular}{|l} 
Dvpmt de \\
logiciels
\end{tabular} & Intégration & $\begin{array}{l}\text { Communi } \\
\text { cation }\end{array}$ & Résultats \\
\hline $\mathrm{C} 1$ & $\begin{array}{l}\text { Elaboration de } \\
\text { graphismes }\end{array}$ & 0,75 & 0,75 & 0 & 0 & 0,25 & 1 \\
\hline $\mathrm{C} 2$ & Intégration de vidéo & 0,25 & 0,25 & 0 & 0 & 0,25 & 0,75 \\
\hline $\mathrm{C} 3$ & $\begin{array}{l}\text { Hébergement de } \\
\text { site }\end{array}$ & 0,75 & 0 & 0,75 & 0 & 0 & 0,75 \\
\hline $\mathrm{C} 4$ & $\begin{array}{l}\text { Programmation } \\
\text { internet }\end{array}$ & 1 & 0 & 1 & 0 & 0 & 0,75 \\
\hline $\mathrm{C} 5$ & $\begin{array}{l}\text { Maîtrise système } \\
\text { d'exploitation }\end{array}$ & 1 & 0 & 1 & 0,25 & 0 & 0,5 \\
\hline C6 & $\begin{array}{l}\text { Développement de } \\
\text { base de données }\end{array}$ & 1 & 0 & 1 & 0 & 0 & 0,5 \\
\hline $\mathrm{C} 7$ & $\begin{array}{l}\text { Réalisation de CD } \\
\text { ROM }\end{array}$ & 0,5 & 0 & 0,5 & 0 & 0,25 & 0,25 \\
\hline $\mathrm{C} 8$ & $\begin{array}{l}\text { Intégration } \\
\text { d'applications }\end{array}$ & 1 & 0 & 0 & 1 & 0 & 0,5 \\
\hline C9 & $\begin{array}{l}\text { Identification des } \\
\text { besoins clients }\end{array}$ & 1 & 0,5 & 0,75 & 0,75 & 1 & 0,25 \\
\hline C10 & $\begin{array}{l}\text { Réalisation de } \\
\text { maquettes }\end{array}$ & 0,5 & 0 & 0 & 0 & 0,5 & 0,5 \\
\hline
\end{tabular}

Tableau 4 : Synthèse de l'audit des compétences

\subsubsection{Evaluation de l'efficacité}

Nous utilisons la formule [1] pour calculer la distance entre le sous-ensemble objectif et le sous-ensemble résultat: $d_{1}(\tilde{R}(E), \tilde{O}(E))=0,35$, puis la formule [3] pour calculer la valeur de l'efficacité : $\varepsilon_{1}=1-d_{1}(\tilde{R}(E), \tilde{O}(E))=0,65$

Nous déterminons ensuite par la formule [5] l'intersection entre le sous-ensemble objectif et le sous-ensemble résultat afin de calculer l'efficacité $\varepsilon_{1}^{\prime}$ excluant l'écart dû aux sur-compétences. Le résultat de cette intersection, ainsi que les intersections $\tilde{R}_{j}\left(\right.$ E) entre le sous-ensemble résultat $\tilde{R}(\mathrm{E})$ et chaque objectif $\tilde{M}_{j}(\mathrm{E})$ de macrocompétences sont donnés à titre d'exemple pour la compétence $\mathrm{C} 1$ :

\begin{tabular}{|c|c|c|c|c|c|}
\hline & $\tilde{R}(E) \cap \tilde{O}(E)$ & $\tilde{R}(E) \cap \tilde{M}_{1}(E$ & $\tilde{R}(E) \cap \tilde{M}_{2}\left(E \tilde{R}(E) \cap \tilde{M}_{3}(E\right.$ & $\tilde{R}(E) \cap \tilde{M}_{4}(E$ \\
\hline $\mathrm{C} 1$ & 0,75 & 0,75 & 0 & 0 & 0,25 \\
\hline
\end{tabular}

Tableau 5 : Intersections entre objectifs et résultats 
Nous obtenons alors $d_{1}(\tilde{R}(E) \cap \tilde{O}(E), \tilde{O}(E))=0,275$

et $\varepsilon_{1}^{\prime}=1-d_{1}(\tilde{R}(E) \cap \tilde{O}(E), \tilde{O}(E))=0,725$

Pour mesurer l'efficacité par objectif de macro-compétences, nous utilisons les formules [12] et [13] :

$$
\begin{aligned}
& d_{3}\left(\tilde{R}_{1}(E), \tilde{M}_{1}(E)\right)=0,25\left(n_{1}=3\right), d_{3}\left(\tilde{R}_{2}(E), \tilde{M}_{2}(E)\right)=2\left(n_{2}=6\right), \\
& d_{3}\left(\tilde{R}_{3}(E), \tilde{M}_{3}(E)\right)=1\left(n_{3}=3\right), d_{3}\left(\tilde{R}_{4}(E), \tilde{M}_{4}(E)\right)=0,75\left(n_{4}=5\right) . \\
& \varepsilon_{3,1}^{\prime}=1-\frac{d_{3}\left(\tilde{R}_{1}(E), \tilde{M}_{1}(E)\right)}{n_{1}}=0,92 ; \varepsilon_{3,2}^{\prime}=0,67 ; \varepsilon_{3,3}^{\prime}=0,67 ; \varepsilon_{3,4}^{\prime}=0,85 .
\end{aligned}
$$

\subsubsection{Evaluation de la pertinence et de l'efficience}

Dans le cadre de cet exemple, nous n'avons instancié qu'une compétence $\mathrm{C}_{\mathrm{i}, \mathrm{j}, \mathrm{k}}$ par compétence type $\mathrm{C}_{\mathrm{i}}$, de sorte que la fonction d'agrégation de la formule [15] se ramène à une fonction identité : $\mu_{\mathrm{N}(\mathrm{E})}\left(\mathrm{C}_{\mathrm{i}}\right)=\mu\left(\mathrm{C}_{\mathrm{i}, \mathrm{j}, \mathrm{k}}\right)$. La caractérisation des moyens et la détermination du niveau de compétence théorique à partir de la formule [15] sont regroupées dans le tableau suivant :

\begin{tabular}{|c|c|c|c|c|}
\hline Compétence & Situation & Acteur & Ressource & $\tilde{N}(E)$ \\
\hline C1 & 0,75 & 0,75 & 1 & 0,75 \\
\hline C2 & 0,25 & 0,75 & 0,75 & 0,25 \\
\hline C3 & 0,75 & 0,75 & 1 & 0,75 \\
\hline C4 & 1 & 1 & 1 & 1 \\
\hline C5 & 0,5 & 0,75 & 1 & 0,5 \\
\hline C6 & 0,75 & 0,75 & 1 & 0,75 \\
\hline C7 & 0,75 & 0,75 & 0,5 & 0,5 \\
\hline C8 & 0,5 & 0,75 & 1 & 0,5 \\
\hline C10 & 0,75 & 0,75 & 1 & 0,75 \\
\hline
\end{tabular}

Tableau 6 : Détermination du niveau de compétence théorique

Lors de l'étude, les évaluations respectives du degré de maîtrise pour la situation, de l'adéquation des acteurs et enfin du niveau de service des ressources ont été réalisées sur des échelles qualitatives à cinq niveaux. Ces échelles sont précisées dans le tableau ci-dessous. Elles intègrent à la fois une dimension qualitative (capacités des acteurs et capabilités des ressources) et une dimension quantitative (liée à la disponibilité). 


\begin{tabular}{|c|c|c|c|}
\hline & situation & acteur & ressource \\
\hline 0 & $\begin{array}{l}\text { Situation professionnelle non } \\
\text { maîtrisée }\end{array}$ & $\begin{array}{l}\text { Capacités inadéquates ou } \\
\text { acteur non disponible }\end{array}$ & $\begin{array}{l}\text { Capabilité inadaptée ou } \\
\text { ressource indisponible }\end{array}$ \\
\hline 0,25 & $\begin{array}{l}\text { Situation faiblement analysée } \\
\text { et rationalisée }\end{array}$ & $\begin{array}{l}\text { Capacités faiblement } \\
\text { adéquates }\end{array}$ & Capabilité peu adaptée \\
\hline 0,5 & $\begin{array}{l}\text { Situation faiblement explicitée } \\
\text { mais maîtrisée par du savoir- } \\
\text { faire }\end{array}$ & $\begin{array}{l}\text { Capacités adéquates et faible } \\
\text { disponibilité }\end{array}$ & $\begin{array}{l}\text { Capabilité adaptée et faible } \\
\text { disponibilité }\end{array}$ \\
\hline 0,75 & $\begin{array}{l}\text { Situation contrôlée par des } \\
\text { indicateurs de performance }\end{array}$ & $\begin{array}{l}\text { Capacités adéquates et bonne } \\
\text { disponibilité }\end{array}$ & $\begin{array}{l}\text { Capabilité adaptée et bonne } \\
\text { disponibilité }\end{array}$ \\
\hline 1 & Situation optimisée & $\begin{array}{l}\text { Capacités adéquates et } \\
\text { excellente disponibilité }\end{array}$ & $\begin{array}{l}\text { Capabilité adaptée et } \\
\text { excellente disponibilité }\end{array}$ \\
\hline
\end{tabular}

Tableau 7 : Echelles de valeur qualitatives

Nous déterminons alors par les formules [17] et [18] la distance entre l'objectif visé et le niveau de compétence théorique, puis la pertinence :

$$
d_{1}(\tilde{N}(E), \tilde{O}(E))=0,175 \text {, et } \rho_{1}=0,825
$$

La formule [19] donne la pertinence après extraction de l'excédent de moyens :

$$
d_{1}(\tilde{N}(E) \cap \tilde{O}(E), \tilde{O}(E))=0,15 \text {, et } \rho_{1}^{\prime}=0,85
$$

Ce qui donne un excédent de moyens par rapport au standard du secteur de $\rho_{1-}^{\prime} \rho_{1}=0,025$.

Les formules [20] et [21] donnent la pertinence par macro-compétences :

$$
\begin{array}{lll}
d_{3}\left(\tilde{N}_{1}(E), \tilde{M}_{1}(E)\right)=0 & \text { avec } n_{1}=3 & \text { et } \rho_{3,1}^{\prime}=1 \\
d_{3}\left(\tilde{N}_{2}(E), \tilde{M}_{2}(E)\right)=0,5 & \text { avec } n_{2}=6 & \text { et } \rho_{3,2}^{\prime}=0,88 \\
d_{3}\left(\tilde{N}_{3}(E), \tilde{M}_{3}(E)\right)=0,5 & \text { avec } n_{3}=3 & \text { et } \rho_{3,3}^{\prime}=0,83 \\
d_{3}\left(\tilde{N}_{4}(E), \tilde{M}_{4}(E)\right)=0,25 & \text { avec } n_{4}=5 & \text { et } \rho_{3,4}^{\prime}=0,95
\end{array}
$$

Pour le calcul de l'efficience, nous vérifions que $\tilde{R}(E) \subseteq \tilde{N}(E)$, puis nous appliquons les formules [22] et [23] : $d_{1}(\tilde{N}(E), \tilde{R}(E))=0,225$, et $\eta_{1}=0,775$.

\subsection{Synthèse de l'étude de cas}

Les calculs que nous venons de développer visent à fournir des outils opérationnels pour piloter le développement des compétences dans une organisation. Ainsi l'étude de cas débouche principalement sur deux outils : d'une part un tableau 
de bord de performances orientées sur la compétence ; et d'autre part un tableau d'identification et de classement des actions de développement des compétences.

Le tableau suivant résume les indicateurs de performance calculés précédemment. Il constitue un tableau de bord synthétique pour le pilotage du système de gestion des compétences de l'entreprise.

\begin{tabular}{|l|c|l|}
\hline Efficacité globale (Ecart résultats / objectifs) & $\varepsilon_{1}$ & $65 \%$ \\
\hline Efficacité hors sur-qualité & $\varepsilon_{1}^{\prime}$ & $72,5 \%$ \\
\hline Efficacité par rapport à la macrocompétence $\mathrm{M}_{1}$ & $\varepsilon_{3,1}$ & $92 \%$ \\
\hline Efficacité par rapport à la macrocompétence $\mathrm{M}_{2}$ & $\varepsilon_{3,2}^{\prime}$ & $67 \%$ \\
\hline Efficacité par rapport à la macrocompétence $\mathrm{M}_{3}$ & $\varepsilon_{3,3}^{\prime}$ & $67 \%$ \\
\hline Efficacité par rapport à la macrocompétence $\mathrm{M}_{4}$ & $\varepsilon_{3.4}^{\prime}$ & $85 \%$ \\
\hline Pertinence (Ecart moyens / objectifs) & $\rho_{1}$ & $82,5 \%$ \\
\hline Pertinence par rapport à la macrocompétence $\mathrm{M}_{1}$ & $\rho_{3,1}^{\prime}$ & $100 \%$ \\
\hline Pertinence par rapport à la macrocompétence $\mathrm{M}_{2}$ & $\rho_{3,2}^{\prime}$ & $88 \%$ \\
\hline Pertinence par rapport à la macrocompétence $\mathrm{M}_{3}$ & $\rho_{3.3}^{\prime}$ & $83 \%$ \\
\hline Pertinence par rapport à la macrocompétence $\mathrm{M}_{4}$ & $\rho_{3,4}^{\prime}$ & $95 \%$ \\
\hline Excédent de moyens & $\rho_{1}^{\prime}-\rho_{1}$ & $2,5 \%$ \\
\hline Efficience (Ecart résultats / moyens) & $\eta_{1}$ & $77,5 \%$ \\
\hline
\end{tabular}

Tableau 8 : Tableau de bord pour le pilotage du système de compétences

Ce tableau de bord fournit une vision agrégée des performances du système de compétences d'une organisation. On peut en particulier y distinguer les non performances dues à des facteurs d'efficience et celles dues à des facteurs de pertinence. Un premier niveau d'analyse causale peut être réalisé pour identifier les compétences de base à l'origine d'une non performance globale, ou bien à l'origine d'une non performance sur une macro-compétence donnée.

Cette analyse causale permettra bien entendu de classer par priorités les différentes actions potentielles de développement des compétences. Ainsi dans le tableau suivant les dix compétences de base sont classées selon leur impact sur une non performance en efficacité. Les différents niveaux de gris soulignent les sources de non efficience ou de non pertinence majeures du système de compétences.

\begin{tabular}{|l|l|l|l|l|l|l|l|l|l|l|}
\hline \multicolumn{1}{|c|}{ (Unité : \%) } & C9 & C2 & C5 & C8 & C6 & C1 & C4 & C7 & C10 & C3 \\
\hline Non efficacité sur $\mathrm{C}_{\mathrm{i}}$ & $\mathbf{7 5}$ & $\mathbf{5 0}$ & $\mathbf{5 0}$ & $\mathbf{5 0}$ & $\mathbf{5 0}$ & $\mathbf{2 5}$ & $\mathbf{2 5}$ & $\mathbf{2 5}$ & $\mathbf{0}$ & $\mathbf{0}$ \\
\hline Sur-compétence sur $\mathrm{C}_{\mathrm{i}}$ & $\mathbf{0}$ & $\mathbf{0}$ & $\mathbf{0}$ & $\mathbf{0}$ & $\mathbf{0}$ & $\mathbf{0}$ & $\mathbf{0}$ & $\mathbf{0}$ & $\mathbf{2 5}$ & $\mathbf{0}$ \\
\hline Non pertinence sur $\mathrm{C}_{\mathrm{i}}$ & $\mathbf{2 5}$ & $\mathbf{0}$ & $\mathbf{5 0}$ & $\mathbf{5 0}$ & $\mathbf{2 5}$ & $\mathbf{0}$ & $\mathbf{0}$ & $\mathbf{0}$ & $\mathbf{0}$ & $\mathbf{0}$ \\
\hline Non efficience sur $\mathrm{C}_{\mathrm{i}}$ & $\mathbf{5 0}$ & $\mathbf{5 0}$ & $\mathbf{0}$ & $\mathbf{0}$ & $\mathbf{2 5}$ & $\mathbf{2 5}$ & $\mathbf{2 5}$ & $\mathbf{2 5}$ & $\mathbf{2 5}$ & $\mathbf{0}$ \\
\hline
\end{tabular}

Tableau 9 : Classement des sources de non performance

Lorsque la pertinence des compétences est en cause, on peut agir sur les leviers d'action proposés au paragraphe 5.3. En cas de non efficience sur une compétence $C_{i}$ 
une analyse causale complémentaire doit être développée pour identifier les interactions entre la situation, les acteurs et les ressources à améliorer (Cf. leviers d'action proposés au paragraphe 5.4).

\section{Conclusions : limites et perspectives}

Nous souhaiterions dans cette conclusion préciser les limites des modèles proposés en vue du pilotage des compétences en enreprise, les perspectives qui s'en dégagent ainsi que le domaine d'application actuel. Nous avons cherché à démontrer dans cet article l'intérêt du modèle s-a-r-C. Cependant il est clair que ce modèle de la compétence n'a pas de valeur ontologique. Il est construit en référence à une définition structurée du concept de compétence (\$3.2) résultant de la synthèse de travaux scientifiques majoritairement issus de la sociologie, des sciences de gestion et du génie industriel. Plus que sa valeur de vérité, l'enjeu de ce modèle est avant tout sa valeur de pilotage : il vise à permettre d'intervenir en entreprise de manière pertinente, en synthétisant une vision suffisante (à un niveau de décision donné), cohérente et constructive d'un certain nombre de variables d'action.

Ainsi, d'autres modèles alternatifs pourront être développés ou sont d'ores et déjà en cours de développement. C'est par exemple le cas des modèles développés par [DUL 01] qui proposent une description structurelle de la compétence plus fine que le modèle s-a-r-C, en se référant à la notion cognitive de schème. Cependant l'objectif de ces modèles est différent : il s'agit de descendre à un niveau de pilotage de la compétence plus fin. Certaines des variables d'action proposées peuvent ainsi être rapprochées des facteurs d'efficience ou de pertinence que nous avons commencé à identifier ci-dessus. C'est donc la complémentarité de ces modèles alternatifs qui en fera l'intérêt.

En ce qui concerne le modèle représentant les macro-compétences d'une organisation, nous avons souligné l'intérêt de son caractère ensembliste (théorie des sous-ensembles flous). Cependant il requiert certains approfondissements afin de mieux définir les mécanismes d'agrégation nécessaires, notamment en références aux travaux existant sur l'utilisation de la théorie des sous-ensembles flous appliquée à la performance [BER 97]. Typiquement, pour la construction d'un niveau de compétence théorique $(§ 5.2)$, nous avons utilisé une fonction de variables floues . Dans l'étude de cas, cet fonction a été assimilée à l'opérateur logique flou «et», mais comme nous l'avons souligné ci-dessus cette fonction nécessite d'être affinée, par exemple par une démarche d'étalonnage lié à un secteur d'activité donné.

Enfin, nous avons indiqué que l'étude de cas traitée dans cet article concerne un groupement coopératif de PME. Le domaine d'application de l'entreprise distribuée, qui repose sur une vision dynamique de l'organisation nous semble en effet particulièrement demandeur de ces nouvelles perspectives sur le pilotage. Le pilotage par les compétences a d'ailleurs déjà fait l'objet de plusieurs travaux (en particulier [HAL 00], [GEN 02], [HAM 02]). Dans ce prolongement, les recherches actuelles de notre équipe s'orientent vers l'élaboration de cartographies dynamiques de compétences pour les groupements d'entreprises. 


\section{Bibliographie}

[AMH 00] AMHERdt CH., DuPuich-RABASSE F., EMERy, GIAUQue, « Compétences collectives dans les organisations », Presses Universitaires de Laval, 2000.

[Bes 95] Bescos P. L., Dobler P., Mendoza C., Naulleau G., « Contrôle de gestion et Management », Editions Montchrestien, Collection Entreprendre, Guide des techniques et de la décision, Paris, 1995.

[BER 97] BERRAH L., «Une approche d'évaluation de la performance industrielle : Modèle d'indicateur et technique floues pour un pilotage réactif », Thèse de l'INPG, 1997.

[Ber 01] Berrah L., Cliville V., Harzallah M., Haurat A., Vernadat F., « Une démarche cyclique d'amélioration permanente pour la réorganisation de systèmes industriels », Actes du $4^{\circ}$ Congrès International de Génie Industriel, 12-15 Juin 2001.

[BOU 99] BOUCHER X., « Contribution méthodologique pour la gestion de filières métiers dans un contexte d'Ingénierie Concourante », Th. Université Aix-Marseille III, Sept 99.

[Bou 01] Boucher X., Harzallah M., «Intégration de la gestion des compétences dans le génie industriel : acquis et perspectives », $4^{\circ}$ Congrès International de Génie Industriel, Marseille, 12-15 Juin 2001.

[Bou 02] Boullet O., Daudonnet M., Beille F., « Le projet ARIBAT », rapport de projet industriel, sous la direction de X. Boucher, Ecole Nationale Supérieure des Mines de St Etienne, Mars 2002.

[Bot 00] Le Boterf G., « Construire les compétences individuelles et collectives », Ed d'Organisation, 2000.

[Boy 82] R.E. Boyatzis, « The Competent manager : a model for effective performance », Ed. Hardcover, 1982

[Dul 01] Dulmet MY., Bonjour E., Lhote F., Mercier G., « Modèle de caractérisation interne des compétences mises en œuvre dans l'entreprise », $4^{\circ}$ Congrès International de Génie Industriel, Marseille, 12-15 Juin 2001

[GAL 90] GaLlois P. M., «Evaluation et pilotage de la performance industrielle » in Ecosip, Gestion Industrielle et Mesure Economique, Economica, pp. 275-294, 1990.

[FRA 00] FRANCHINI L., « Aide à la décision pour la gestion des opérateurs en production : Modélisation, Planification et Elaboration », thèse de l'INP-Toulouse, novembre 2000.

[GAR 99] GARDONI M., «Maîtrise de l'information non structurée et capitalisation de savoir et savoir-faire en Ingénierie Intégrée. Cas d'étude Aérospatiale », thèse de l'Université de Metz, juillet 1999.

[Gen 02] Geniaux I., Bonnardel S., « Gestion des compétences et Knowledge Management dans les réseaux d'entreprises », Colloque Gestion des Compétences et Knowledge Management : renouveau de création de valeur en GRH?, Rouen, mars 2002.

[GRA 00] GRABOT B., LetOUZEY A., « Short-term manpower management in manufacturing systems : new requirements and DSS prototyping », Computers in Industry, Vol.43, $\mathrm{N}^{\circ} 1$, pp.11-29, 2000.

[Hal 00] Halley A., Beaulieu M., « La métamorphose des compétences en contexte d'intégration de la chaîne d'approvisionnement », Actes des troisièmes Rencontres Internationales de la recherche en Logistique, Trois-Rivières, Canada, 9 au 11 mai 2000. 
[Ham 02] Hammami A., Burlat P., Boucher X., « Distributed enterprises configuration : orders allocation within Networks of Firms », 3rd IFIP Working Conference on Infrastructures for Virtual Enterprises, Sesimbra, Portugal, 1-3 May 2002.

[HAR 00] HaRzallah M., «Modélisation des aspects organisationnels et des compétences pour la réorganisation d'entreprises industrielles », Thèse Université de Metz, mai 2000.

[JIA 98] JIA T., «Vers une meilleure gestion des ressources d'un groupe autonome de fabrication », thèse de l'université de Tours, décembre 1998.

[LEV 96] LEVY-LEBOYER C., «La gestion des compétences », Eds de l'organisation, 1996.

[LOR 97] LORINO P., «Méthodes et pratiques de la performance : le guide du pilotage », Paris, Editions d'Organisation, 1997.

[LON 95] NONAKA I., TAKEUCHI H., « The knowledge creating company », Oxford University Press, 1995.

[KAP 98] KAPLAN R.S. NORTON D.P., «Le tableau de bord prospectif. Pilotage stratégique : les quatre axes de succès », Ed. d'organisation, Paris, 1998.

[PER 00] PERVAIZ K.A., ZAIRI M. «Innovation : a performance Measurement perspective » dans TIDD J., From Knowledge Management to Strategic Competence. Measuring technological, market and organizational innovation, Imperial College Press 2000.

[POR 93] PORTER M. E., «L'avantage concurrentiel des nations », Paris, InterEds, 1993.

[PRA 92] PRAHALAD CK., HAMEL G., CThe core competence of the corporation », IEEE Engineering Management Review, fall 1992, pp5-14.

[Que 00] Quelin B. , ARREGLE J. L. , « Le management stratégique des compétences », Editions ellipses, 2000.

[RAU 01] RAUCH-GEelHAAR C., WARNECKE G., «Innovation based on life cycle engineering and knowledge management », Revue Internationale d'Ingénierie des Systèmes de Production Mécanique, n5, juin 2001.

[SAN 96] Sanchez R., HeEne A., Thomas H., « Dynamics of Competence-based Competition », Elsevier Science Ltd., 1996.

[Son 00] Sonntag M., OuKacha M., «Implication des acteurs et gestion des informations et

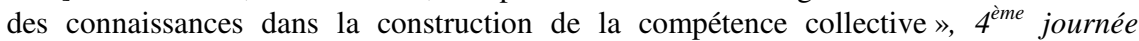
d'étude sur la gestion des compétences et des connaissances en génie industriel. Saint Etienne, Novembre 2000.

[SvE 97], Sveiby K., 2001, «The Intangible Asset Monitor », Journal of Human Resources and Cost Accounting, vol $2 \mathrm{n}^{\circ} 1$, pp 73-97, spring 97.

[Sze 00] Szegheo O., Petersen S.A., «Extended Enterprise Engineering - A Model-based Framework », Journal of Concurrent Engineering Research and Applications , Vol. 8, No. 1, March 2000, pp 32.

[Ter 96] De TerssaC G., « Savoir, Compétences et travail », Rapport 96225, juin 1996.

[Ver 99] Vernadat F., "Technique de Modélisation en Entreprise : Applications aux processus opérationnels », Economica, 1999.

[ZAD 65] ZADEH L. A., « Fuzzy sets », Information and Control, vol. 8, pp. 338-353, 1965.

[ZAR 01] ZARIFIAN P., "Le modèle de la compétence. Trajectoire historique, enjeux actuels et propositions », Editions Liaisons, 2001. 